\title{
Variable bandwidth local maximum likelihood type estimation for diffusion processes
}

\author{
Ming T. Tang and Yun Y. Wang* (D)
}

\section{"Correspondence:} yywang@zju.edu.cn School of Science, Jiangxi University of Science and Technology, Ganzhou, People's Republic of China

\begin{abstract}
The method of robust approach is applied to estimate drift function and diffusion function of diffusion processes with discrete-time observations. The proposed method combines the ideas of local linear regression technique and maximum likelihood type estimation technique, so the advantages of local linear estimators persist and overcome the disadvantages of least-squares estimator. Moreover, a variable bandwidth instead of a constant bandwidth is considered in the local maximum likelihood type estimators. The consistency and asymptotic normality of the local maximum likelihood type estimators for drift and diffusion functions are developed under some given conditions. We perform a simulation study to evaluate the robust performances of the proposed estimators.
\end{abstract}

MSC: $62 \mathrm{G} 35 ; 62 \mathrm{G} 20$

Keywords: Asymptotic normality; Consistency; Robust estimation; Stochastic differential equation; Variable bandwidth

\section{Introduction}

Diffusion processes $X$ defined by the following stochastic differential equation are considered in this article:

$$
d X_{t}=\mu\left(X_{t}\right) d t+\sigma\left(X_{t}\right) d B_{t}
$$

where $\left\{B_{t}, t \geq 0\right\}$ is a standard Brownian motion, $\mu(\cdot)$ is an unknown measurable function (drift function) and $\sigma(\cdot)$ is an unknown positive function (diffusion function). It is well known that diffusion processes driven by Brown motion have been widely used in the financial and economic fields, and it is often used to model and analyze dynamic changes in asset prices, interest rates, and exchange rates. For example, [1] investigated an application of Ornstein-Uhlenbeck process to commodity pricing in Thailand. Reference [2] improved estimation of drift parameters of diffusion processes for interest rates by incorporating information in bond prices. So, recently in the literature, the statistical inference for diffusion processes based on discrete observations has often been of concern; for example, see [3-6] and its references for parametric estimation, see [7-9] and the references

(c) The Author(s) 2018. This article is distributed under the terms of the Creative Commons Attribution 4.0 International License (http://creativecommons.org/licenses/by/4.0/), which permits unrestricted use, distribution, and reproduction in any medium, provided you give appropriate credit to the original author(s) and the source, provide a link to the Creative Commons license, and indicate if changes were made. 
therein for a semi-parametric estimation and see [10-16] and the references therein for a nonparametric estimation.

As is well known, the first to consider nonparametric estimation for the diffusion coefficient in model (1) with discrete-time observation was [17], where a kernel type estimator was considered. Thereafter, [18] proposed a nonparametric identification and an estimation procedure for the diffusion function after [17], and derived a consistent nonparametric estimator for the drift function by combining their estimator of the diffusion function. Reference [19] constructed the first-, second-, and third-order approximation formulas for drift and diffusion functions by using an infinitesimal generator and Taylor expansion. Reference [12] generalized Stanton's idea and introduced the local polynomial estimators for drift and diffusion functions. Since a local linear method may produce negative values for the diffusion function, [14] proposed a new nonparametric estimation procedure of the diffusion function based on re-weighting the Nadaraya-Watson estimator.

However, local linear regression methods are very sensitive to outliers, and individual outliers can lead to large changes in the results of statistical inference, therefore leading to irrational and even erroneous conclusions. Such statistical methods like the local linear regression approach are not strong enough to adapt the complex changing reality, in other words, the local linear regression method is not robust when it comes to outliers or heavy-tailed distributions. In recent years, various robust methods have been proposed for abnormal observation, which has become increasingly crucial and frequent in many research fields. Reference [20] defined maximum likelihood type robust estimates of regression and investigated the asymptotic properties. From then on, the maximum likelihood type robust estimation (M-estimation) has been discussed by many authors, for example, $[21,22]$ and the references therein. Meanwhile, some modified maximum likelihood type estimators were developed, such as the local maximum likelihood type estimator (local M-estimators), which is a combination of the local linear regression and the $\mathrm{M}$-estimation regression, so the nice properties of local linear estimator and M-estimator persist. For instance, [23] constructed variable bandwidth local linear M-estimator for a regression function. Reference [24] proposed a nonparametric estimator of the regression function by combining local polynomial regression and M-estimation regression. Reference [25] developed robust version of local linear regression smoothers for stationary time series sequence. Reference [26] considered local M-estimation of the unknown drift and diffusion functions of integrated diffusion processes.

The purpose of this paper is to investigate the local linear and variable bandwidth Mestimators of the drift and diffusion functions in model (1) based on high-frequency data, that is, the sample observations are only selected at discrete-time points, say at $n$ equally spaced $\{i \Delta, i=0,1, \ldots, n\}$, where $\Delta$ is the sampling interval, and $\Delta \rightarrow 0$ as $n \rightarrow \infty$. Furthermore, the consistency and asymptotic normality of the local M-estimators will be proved under general assumptions. Since the new the local linear and variable bandwidth M-estimators have a good control of outliers, which are common in financial, economic, physical, engineering and other fields, the proposed estimators in this paper greatly promote the application of diffusion model (1) in these fields, and they provide the theory and application foundation for dynamic modeling in these fields. 
The article is organized as follows. The second section constructs the variable bandwidth local M-estimators of drift and diffusion functions, and the consistency and asymptotic normality of the new estimators are developed in the same section. Section 3 presents the results of a simulation study. Proofs and auxiliary results are given in Section 4.

\section{Local M-estimators and asymptotic theory}

Local M-estimation of drift function $\mu(x)$ and diffusion function $\sigma^{2}(x)$ depend on the equations

$$
\begin{aligned}
& E\left(\frac{X_{(i+1) \Delta}-X_{i \Delta}}{\Delta} \mid X_{i \Delta}=x\right)=\mu(x)+o(1), \\
& E\left(\frac{\left(X_{(i+1) \Delta}-X_{i \Delta}\right)^{2}}{\Delta} \mid X_{i \Delta}=x\right)=\sigma^{2}(x)+o(1),
\end{aligned}
$$

as $\Delta \rightarrow 0$. The reader can refer to [19] or [27] for more details as regards (2) and (3).

Neglecting the smaller-order terms, the local linear estimator with the variable bandwidth for $\mu(x)$ is defined as the solution to the problem: Choose $a_{1}$ and $b_{1}$ to minimize the weighted sum as

$$
\sum_{i=1}^{n}\left(\frac{X_{(i+1) \Delta}-X_{i \Delta}}{\Delta}-a_{1}-b_{1}\left(X_{i \Delta}-x\right)\right)^{2} \beta_{1}\left(X_{i \Delta}\right) K\left(\frac{X_{i \Delta}-x}{h} \beta_{1}\left(X_{i \Delta}\right)\right),
$$

and the local linear estimator with the variable bandwidth for $\sigma^{2}(x)$ is defined as the solution to the problem: Choose $a_{2}$ and $b_{2}$ to minimize the weighted sum as follows:

$$
\sum_{i=1}^{n}\left(\frac{\left(X_{(i+1) \Delta}-X_{i \Delta}\right)^{2}}{\Delta}-a_{2}-b_{2}\left(X_{i \Delta}-x\right)\right)^{2} \beta_{2}\left(X_{i \Delta}\right) K\left(\frac{X_{i \Delta}-x}{h} \beta_{2}\left(X_{i \Delta}\right)\right),
$$

where $K(\cdot)$ is kernel function and $h=h_{n}$ is the bandwidth. $\beta_{1}(\cdot)$ and $\beta_{2}(\cdot)$ are nonnegative functions reflecting the variable amount of smoothing at each data point. $h / \beta_{1}\left(X_{i \Delta}\right)$ and $h / \beta_{2}\left(X_{i \Delta}\right)$ are called variable bandwidth. For more detailed information on variable bandwidths, see [28-30], among others.

In fact, the aforementioned method used to establish estimators are based on leastsquares approach and are not robust. As a result, we choose $a_{1}$ and $b_{1}$ to minimize

$$
\sum_{i=1}^{n} \rho_{1}\left(\frac{X_{(i+1) \Delta}-X_{i \Delta}}{\Delta}-a_{1}-b_{1}\left(X_{i \Delta}-x\right)\right) \beta_{1}\left(X_{i \Delta}\right) K\left(\frac{X_{i \Delta}-x}{h} \beta_{1}\left(X_{i \Delta}\right)\right)
$$

and $a_{2}$ and $b_{2}$ to minimize

$$
\sum_{i=1}^{n} \rho_{2}\left(\frac{\left(X_{(i+1) \Delta}-X_{i \Delta}\right)^{2}}{\Delta}-a_{2}-b_{2}\left(X_{i \Delta}-x\right)\right) \beta_{2}\left(X_{i \Delta}\right) K\left(\frac{X_{i \Delta}-x}{h} \beta_{2}\left(X_{i \Delta}\right)\right)
$$

or to satisfy the following estimation equations:

$$
\sum_{i=1}^{n} \psi_{1}\left(\frac{X_{(i+1) \Delta}-X_{i \Delta}}{\Delta}-a_{1}-b_{1}\left(X_{i \Delta}-x\right)\right) \beta_{1}\left(X_{i \Delta}\right) K\left(\frac{X_{i \Delta}-x}{h / \beta_{1}\left(X_{i \Delta}\right)}\right)\left(\begin{array}{c}
1 \\
\frac{X_{i \Delta}-x}{h}
\end{array}\right)=\left(\begin{array}{l}
0 \\
0
\end{array}\right)
$$


and

$$
\begin{aligned}
& \sum_{i=1}^{n} \psi_{2}\left(\frac{\left(X_{(i+1) \Delta}-X_{i \Delta}\right)^{2}}{\Delta}-a_{2}-b_{2}\left(X_{i \Delta}-x\right)\right) \beta_{2}\left(X_{i \Delta}\right) K\left(\frac{X_{i \Delta}-x}{h / \beta_{2}\left(X_{i \Delta}\right)}\right)\left(\begin{array}{c}
1 \\
\frac{X_{i \Delta}-x}{h}
\end{array}\right) \\
& \quad=\left(\begin{array}{l}
0 \\
0
\end{array}\right),
\end{aligned}
$$

where $\rho_{1}(\cdot)$ and $\rho_{2}(\cdot)$ are given outlier-resistant functions and $\psi_{1}(\cdot)$ and $\psi_{2}(\cdot)$ are the derivatives of $\rho_{1}(\cdot)$ and $\rho_{2}(\cdot)$, respectively.

The maximum likelihood type estimators of $\mu(x)$ and $\mu^{\prime}(x)$ are denoted $\hat{\mu}(x)=\hat{a}_{1}$ and $\hat{\mu}^{\prime}(x)=\hat{b}_{1}$, which are the solutions of (4), the maximum likelihood type estimators of $\sigma^{2}(x)$ and $\left(\sigma^{2}(x)\right)^{\prime}$ are denoted $\hat{\sigma}^{2}(x)=\hat{a}_{2}$ and $\left(\hat{\sigma}^{2}(x)\right)^{\prime}=\hat{b}_{2}$, which are the solutions of (5).

For a given point $x_{0}$, our lemmas and asymptotic theory results are based on the following conditions.

C 1 ([10])

(i) The initial condition $X_{0} \in L^{2}$ and is considered to be independent of $\left\{B_{t}, t \geq 0\right\}$.

(ii) The unknown functions $\mu(\cdot)$ and $\sigma(\cdot)$ are time-homogeneous and measurable functions on $\mathcal{D}=(l, u)$ with $-\infty \leq l<u \leq \infty$. We also assume the two functions are at least twice continuously differentiable, and satisfy local Lipschitz and growth conditions, that is, for any compact subset $J \subseteq \mathcal{D}$, for all $x, y \in J$, there exist constants $L_{1}$ and $L_{2}$ such that

$$
|\mu(x)-\mu(y)|+|\sigma(x)-\sigma(y)| \leq L_{1}|x-y|
$$

and

$$
|\mu(x)|+|\sigma(x)| \leq L_{2}[1+|x|]
$$

(iii) $\sigma^{2}(\cdot)>0$ on $\mathcal{D}$;

(iv) Let $S(z)=\int_{z_{0}}^{z} \exp \left(\int_{z_{0}}^{y} \frac{-2 \mu(x)}{\sigma^{2}(x)} d x\right) d y, z_{0} \in \mathcal{D}$, suppose $S(z)$ satisfies

$$
\begin{aligned}
& \lim _{z \rightarrow l} S(z)=-\infty, \\
& \lim _{z \rightarrow u} S(z)=\infty .
\end{aligned}
$$

Remark 1 The condition $\mathrm{C} 1$ ensures the existence and uniqueness of a strong solution to model (1), see [31] for details.

\section{2}

(i) $\int_{l}^{u} s(x) d x<\infty$, where $s(x)=2 / S^{\prime}(x) \sigma^{2}(x)$.

(ii) The initial point $X_{0}$ has a stationary distribution $P^{0}$, where $P^{0}$ is the invariant distribution of the process $\left(X_{t}\right)_{t \in[0, \infty)}$. 
Remark 2 The conditions $\mathrm{C} 1$ and $\mathrm{C} 2$ ensure that $X$ is stationary, and from Kolmogorov forward equation we can get the stationary density $p(x)$ of $X$ :

$$
p(x)=\frac{s(x)}{\int_{l}^{u} s(x) d x}=\frac{\xi}{\sigma^{2}(x)} \exp \left\{\int_{z_{0}}^{x} \frac{2 \mu(x)}{\sigma^{2}(x)} d x\right\}
$$

where $z_{0}$ is an arbitrary point inside $\mathcal{D}$ and $\xi$ is a normalizing constant.

C 3 Let $\mathcal{D}=(l, u)$ be the state space of $X$, suppose that

$$
\begin{gathered}
\limsup _{x \rightarrow u}\left(\frac{\mu(x)}{\sigma(x)}-\frac{\sigma^{\prime}(x)}{2}\right)<0, \\
\limsup _{x \rightarrow l}\left(\frac{\mu(x)}{\sigma(x)}-\frac{\sigma^{\prime}(x)}{2}\right)>0 .
\end{gathered}
$$

Moreover, the mixing coefficient $\alpha(k)$ satisfies $\sum_{k \geq 1} k^{a}(\alpha(k))^{\gamma /(2+\gamma)}<\infty$ for some $a>$ $\gamma /(2+\gamma)$, where $\gamma$ is given in the condition C8.

Remark 3 The condition C3 guarantees that the process $X$ is $\alpha$-mixing; see [32] for details.

\section{4}

(i) The kernel function $K(\cdot)$ is a continuous probability density function compactly supported on $[-1,1]$.

(ii) The bandwidth $h$ satisfies $h \rightarrow 0, n h \rightarrow \infty$ and $n h \Delta \rightarrow \infty$ as $n \rightarrow \infty$.

C 5 The density function $p(x)$ of the process $X$ is continuous at the point $x_{0}$, and $p\left(x_{0}\right)>0$. Moreover, the joint density function of $X_{i \Delta}$ and $X_{j \Delta}$ is bounded for all $i, j$.

\section{6}

(i) $\min _{x} \beta_{1}(x)>0$, and $\beta_{1}(\cdot)$ is continuous at the point $x_{0}$;

(ii) $\min _{x} \beta_{2}(x)>0$, and $\beta_{2}(\cdot)$ is continuous at the point $x_{0}$.

\section{7}

(i) $E\left[\psi_{1}\left(u_{i \Delta}\right) \mid X_{i \Delta}=x\right]=o(1)$ with $u_{i \Delta}=\frac{X_{(i+1) \Delta}-X_{i \Delta}}{\Delta}-\mu\left(X_{i \Delta}\right)$;

(ii) $E\left[\psi_{2}\left(v_{i \Delta}\right) \mid X_{i \Delta}=x\right]=o(1)$ with $v_{i \Delta}=\frac{\left(X_{(i+1) \Delta}-X_{i \Delta}\right)^{2}}{\Delta}-\sigma^{2}\left(X_{i \Delta}\right)$.

\section{8}

(i) The function $\psi_{1}(\cdot)$ is continuous and has a derivative $\psi_{1}^{\prime}(\cdot)$ almost everywhere. Additionally, we assume that the following three functions: $E\left[\psi_{1}^{\prime}\left(u_{i \Delta}\right) \mid X_{i \Delta}=x\right]$, $E\left[\psi_{1}^{2}\left(u_{i \Delta}\right) \mid X_{i \Delta}=x\right], E\left[\psi_{1}^{\prime 2}\left(u_{i \Delta}\right) \mid X_{i \Delta}=x\right]$ are all positive for any $x$ and continuous at the point $x_{0}$, and there exists a constant $\gamma>0$ such that $E\left[\left|\psi_{1}\left(u_{i \Delta}\right)\right|^{2+\gamma} \mid X_{i \Delta}=x\right]$, $E\left[\left|\psi_{1}^{\prime}\left(u_{i \Delta}\right)\right|^{2+\gamma} \mid X_{i \Delta}=x\right]$ are bounded in a neighborhood of $x_{0}$.

(ii) The function $\psi_{2}(\cdot)$ is continuous and has a derivative $\psi_{2}^{\prime}(\cdot)$ almost everywhere. Additionally, we assume that the following three functions: $E\left[\psi_{2}^{\prime}\left(v_{i \Delta}\right) \mid X_{i \Delta}=x\right]$, $E\left[\psi_{2}^{2}\left(v_{i \Delta}\right) \mid X_{i \Delta}=x\right], E\left[\psi_{2}^{\prime 2}\left(v_{i \Delta}\right) \mid X_{i \Delta}=x\right]$ are all positive for any $x$ and continuous at the point $x_{0}$, and there exists a constant $\gamma>0$ such that $E\left[\left|\psi_{2}\left(v_{i \Delta}\right)\right|^{2+\gamma} \mid X_{i \Delta}=x\right]$, $E\left[\left|\psi_{2}^{\prime}\left(v_{i \Delta}\right)\right|^{2+\gamma} \mid X_{i \Delta}=x\right]$ are bounded in a neighborhood of $x_{0}$. 


\section{9}

(i) The function $\psi_{1}^{\prime}(\cdot)$ satisfies

$$
E\left[\sup _{|z| \leq \delta}\left|\psi_{1}^{\prime}\left(u_{i \Delta}+z\right)-\psi_{1}^{\prime}\left(u_{i \Delta}\right)\right| \mid X_{i \Delta}=x\right]=o(1)
$$

and

$$
E\left[\sup _{|z| \leq \delta}\left|\psi_{1}\left(u_{i \Delta}+z\right)-\psi_{1}\left(u_{i \Delta}\right)-\psi_{1}^{\prime}\left(u_{i \Delta}\right) z\right| \mid X_{i \Delta}=x\right]=o(\delta),
$$

as $\delta \rightarrow 0$ uniformly in $x$ in a neighborhood of $x_{0}$;

(ii) The function $\psi_{2}^{\prime}(\cdot)$ satisfies

$$
E\left[\sup _{|z| \leq \delta}\left|\psi_{2}^{\prime}\left(v_{i \Delta}+z\right)-\psi_{2}^{\prime}\left(v_{i \Delta}\right)\right| \mid X_{i \Delta}=x\right]=o(1)
$$

and

$$
E\left[\sup _{|z| \leq \delta}\left|\psi_{2}\left(v_{i \Delta}+z\right)-\psi_{2}\left(v_{i \Delta}\right)-\psi_{2}^{\prime}\left(v_{i \Delta}\right) z\right| \mid X_{i \Delta}=x\right]=o(\delta)
$$

as $\delta \rightarrow 0$ uniformly in $x$ in a neighborhood of $x_{0}$.

\section{10}

(i) For any $i, j$, suppose that

$$
\begin{aligned}
& E\left[\psi_{1}^{2}\left(u_{i \Delta}\right)+\psi_{1}^{2}\left(u_{j \Delta}\right) \mid X_{i \Delta}=x, X_{j \Delta}=y\right], \\
& E\left[\psi_{1}^{\prime 2}\left(u_{i \Delta}\right)+\psi_{1}^{\prime 2}\left(u_{j \Delta}\right) \mid X_{i \Delta}=x, X_{j \Delta}=y\right]
\end{aligned}
$$

are bounded in the neighborhood of $x_{0}$;

(ii) For any $i, j$, suppose that

$$
\begin{aligned}
& E\left[\psi_{2}^{2}\left(v_{i \Delta}\right)+\psi_{2}^{2}\left(v_{j \Delta}\right) \mid X_{i \Delta}=x, X_{j \Delta}=y\right], \\
& E\left[\psi_{2}^{\prime 2}\left(v_{i \Delta}\right)+\psi_{2}^{\prime 2}\left(v_{j \Delta}\right) \mid X_{i \Delta}=x, X_{j \Delta}=y\right]
\end{aligned}
$$

are bounded in the neighborhood of $x_{0}$.

Remark 4 In fact, the conditions C7-C10 imposed on $\psi_{1}(\cdot)$ and $\psi_{2}(\cdot)$ are mild and satisfied for many applications, such as Huber's $\psi(\cdot)$ function. For more detailed information on these conditions please refer to [23] or [25].

C 11 Suppose that there exist a sequence of positive integers $q_{n}$ such that $q_{n} \rightarrow \infty, q_{n}=$ $o\left((n h)^{1 / 2}\right)$ and $(n / h)^{1 / 2} \alpha\left(q_{n}\right) \rightarrow 0$ as $n \rightarrow \infty$.

C 12 For $\gamma$ in the condition C8 and all $x$ in a neighborhood of $x_{0}$, there exists $\tau>2+$ $\gamma$ such that the two functions $E\left\{\left|\psi_{1}\left(u_{i \Delta}\right)\right|^{\tau} \mid X_{i \Delta}=x\right\}, E\left\{\left|\psi_{2}\left(v_{i \Delta}\right)\right|^{\tau} \mid X_{i \Delta}=x\right\}$ are bounded. Furthermore, we assume $\alpha(n)=O\left(n^{-\theta}\right)$, where $\theta \geq(2+\gamma) \tau /\{2(\tau-2-\gamma)\}$. 
C 13 Assume $n^{-\gamma / 4} h^{(2+\gamma) / \tau-1-\gamma / 4}=O(1)$, where $\gamma$ is given in the condition C8 and $\tau$ is given in the condition $\mathrm{C} 12$.

Remark 5 The assumptions in condition C3 and C11 on mixing coefficient $\alpha(k)$ is sufficient conditions for mixing coefficient, [25] pointed out this assumptions on mixing coefficient are satisfied given some general conditions. Condition C13 is also satisfied under some weak constraints on $\gamma$ or $\tau$.

Throughout the whole paper, let

$$
\begin{aligned}
& K_{l}=\int K(u) u^{l} d u, \quad J_{l}=\int u^{l} K^{2}(u) d u, \quad \text { for } l \geq 0 . \\
& U_{1}=\left(\begin{array}{cc}
K_{0} & \frac{K_{1}}{\beta_{1}\left(x_{0}\right)} \\
\frac{K_{1}}{\beta_{1}\left(x_{0}\right)} & \frac{K_{2}}{\beta_{1}^{2}\left(x_{0}\right)}
\end{array}\right), \quad V_{1}=\left(\begin{array}{cc}
J_{0} & \frac{J_{1}}{\beta_{1}\left(x_{0}\right)} \\
\frac{J_{1}}{\beta_{1}\left(x_{0}\right)} & \frac{J_{2}}{\beta_{1}^{2}\left(x_{0}\right)}
\end{array}\right), \quad A_{1}=\left(\begin{array}{c}
K_{2} \\
\frac{K_{3}}{\beta_{1}\left(x_{0}\right)}
\end{array}\right), \\
& U_{2}=\left(\begin{array}{cc}
K_{0} & \frac{K_{1}}{\beta_{2}\left(x_{0}\right)} \\
\frac{K_{1}}{\beta_{2}\left(x_{0}\right)} & \frac{K_{2}}{\beta_{2}^{2}\left(x_{0}\right)}
\end{array}\right), \quad V_{2}=\left(\begin{array}{cc}
J_{0} & \frac{J_{1}}{\beta_{2}\left(x_{0}\right)} \\
\frac{J_{1}}{\beta_{2}\left(x_{0}\right)} & \frac{J_{2}}{\beta_{2}^{2}\left(x_{0}\right)}
\end{array}\right), \quad A_{2}=\left(\begin{array}{c}
K_{2} \\
\frac{K_{3}}{\beta_{2}\left(x_{0}\right)}
\end{array}\right), \\
& G_{1}(x)=E\left[\psi_{1}^{\prime}\left(u_{i \Delta}\right) \mid X_{i \Delta}=x\right], \quad G_{2}(x)=E\left[\psi_{1}^{2}\left(u_{i \Delta}\right) \mid X_{i \Delta}=x\right] \\
& G_{3}(x)=E\left[\psi_{1}^{\prime 2}\left(u_{i \Delta}\right) \mid X_{i \Delta}=x\right], \quad H_{1}(x)=E\left[\psi_{2}^{\prime}\left(v_{i \Delta}\right) \mid X_{i \Delta}=x\right] \\
& H_{2}(x)=E\left[\psi_{2}^{2}\left(v_{i \Delta}\right) \mid X_{i \Delta}=x\right], \quad H_{3}(x)=E\left[\psi_{2}^{\prime 2}\left(v_{i \Delta}\right) \mid X_{i \Delta}=x\right] .
\end{aligned}
$$

We now develop the asymptotic theory for the proposed local M-estimators:

Theorem 1 Under the conditions C1-C5 and the conditions (i) of C6-C10, there exist solutions $\hat{\mu}\left(x_{0}\right)$ and $\hat{\mu}^{\prime}\left(x_{0}\right)$ to equations (4) such that

(i)

$$
\left(\begin{array}{c}
\hat{\mu}\left(x_{0}\right)-\mu\left(x_{0}\right) \\
h\left(\hat{\mu}^{\prime}\left(x_{0}\right)-\mu^{\prime}\left(x_{0}\right)\right)
\end{array}\right) \stackrel{P}{\rightarrow} 0, \quad n \rightarrow \infty
$$

(ii) Furthermore, if the conditions C11-C13 hold, then

$$
\sqrt{n h}\left[\left(\begin{array}{c}
\hat{\mu}\left(x_{0}\right)-\mu\left(x_{0}\right) \\
h\left(\hat{\mu}^{\prime}\left(x_{0}\right)-\mu^{\prime}\left(x_{0}\right)\right)
\end{array}\right)-\frac{h^{2} \mu^{\prime \prime}\left(x_{0}\right)}{2 \beta_{1}^{2}\left(x_{0}\right)} U_{1}^{-1} A_{1}\right] \stackrel{D}{\rightarrow} N\left(0, \Sigma_{1}\right),
$$

where

$$
\Sigma_{1}=\frac{G_{2}\left(x_{0}\right) \beta_{1}\left(x_{0}\right)}{G_{1}^{2}\left(x_{0}\right) p\left(x_{0}\right)} U_{1}^{-1} V_{1} U_{1}^{-1}
$$

Theorem 2 Under the conditions C1-C5 and the conditions (ii) of C6-C10, there exist solutions $\hat{\sigma}^{2}\left(x_{0}\right)$ and $\left(\hat{\sigma}^{2}\left(x_{0}\right)\right)^{\prime}$ to equations (5) such that

(i)

$$
\left(\begin{array}{c}
\hat{\sigma}^{2}\left(x_{0}\right)-\sigma^{2}\left(x_{0}\right) \\
h\left(\left(\hat{\sigma}^{2}\left(x_{0}\right)\right)^{\prime}-\left(\sigma^{2}\left(x_{0}\right)\right)^{\prime}\right)
\end{array}\right) \stackrel{P}{\rightarrow} 0, \quad n \rightarrow \infty .
$$


(ii) Furthermore, if the conditions C11-C13 hold, then

$$
\sqrt{n h}\left[\left(\begin{array}{c}
\hat{\sigma}^{2}\left(x_{0}\right)-\sigma^{2}\left(x_{0}\right) \\
h\left[\left(\hat{\sigma}^{2}\left(x_{0}\right)\right)^{\prime}-\left(\sigma^{2}\left(x_{0}\right)\right)^{\prime}\right]
\end{array}\right)-\frac{h^{2}\left(\sigma^{2}\left(x_{0}\right)\right)^{\prime \prime}}{2 \beta_{2}^{2}\left(x_{0}\right)} U_{2}^{-1} A_{2}\right] \stackrel{D}{\rightarrow} N\left(0, \Sigma_{2}\right),
$$

where

$$
\Sigma_{2}=\frac{H_{2}\left(x_{0}\right) \beta_{2}\left(x_{0}\right)}{H_{1}^{2}\left(x_{0}\right) p\left(x_{0}\right)} U_{2}^{-1} V_{2} U_{2}^{-1}
$$

\section{Simulation study}

We now perform a Monte Carlo simulation study to evaluate the finite sample performance of the variable bandwidth local M-estimators by comparing the mean square error (MSE) between them and the Nadaraya-Watson estimators.

We consider the following diffusion process $X$ :

$$
d X_{t}=\left(-X_{t}+0.5 \sqrt{1+X_{t}^{2}}\right) d t+0.1 d B_{t}
$$

for $t \in[0, T]=[0,100]$. Throughout the simulation, we take Huber's function $\psi_{1}(z)=$ $\max \{-c, \min (c, z)\}$ with $c=0.135$. The uniform kernel $K(u)=\frac{1}{2} I(|u| \leq 1)$ is selected as the kernel function, and the bandwidth $h$ is chosen by minimizing the MSE as follows:

$$
\frac{1}{n} \sum_{i=1}^{n}\left(\hat{\mu}\left(x_{i}\right)-\mu\left(x_{i}\right)\right)^{2}
$$

where $\left\{x_{i}, i=1,2, \ldots, n\right\}$ are chosen uniformly to cover the range of sample path of $X_{t}$.

Throughout the study, we use iterative method to obtain $\hat{\mu}(\cdot)$, for any initial value $\hat{\mu}_{0}(x)$, we have

$$
\left(\begin{array}{l}
\hat{\mu}_{t}(x) \\
\hat{\mu}_{t}^{\prime}(x)
\end{array}\right)=\left(\begin{array}{c}
\hat{\mu}_{t-1}(x) \\
\hat{\mu}_{t-1}^{\prime}(x)
\end{array}\right)-\left[W_{n}\left(\hat{\mu}_{t-1}(x), \hat{\mu}_{t-1}^{\prime}(x)\right)\right]^{-1} \Psi_{n}\left(\hat{\mu}_{t-1}(x), \hat{\mu}_{t-1}^{\prime}(x)\right),
$$

where $\hat{\mu}_{t-1}(x)$ and $\hat{\mu}_{t-1}^{\prime}(x)$ are the $t$ th iteration value of $\hat{\mu}^{\prime}(x)$ and $\hat{\mu}(x)$, and

$$
\begin{aligned}
W_{n}\left(a_{1}, b_{1}\right)= & \left(\frac{\partial \Psi_{n}\left(a_{1}, b_{1}\right)}{\partial a_{1}}, \frac{\partial \Psi_{n}\left(a_{1}, b_{1}\right)}{\partial b_{1}}\right) \\
\Psi_{n}\left(a_{1}, b_{1}\right)= & \sum_{i=1}^{n} \psi_{1}\left(\frac{X_{(i+1) \Delta}-X_{i \Delta}}{\Delta}-a_{1}-b_{1}\left(X_{i \Delta}-x\right)\right) \beta_{1}\left(X_{i \Delta}\right) \\
& \times K\left(\frac{X_{i \Delta}-x}{h / \beta_{1}\left(X_{i \Delta}\right)}\right)\left(\begin{array}{c}
1 \\
\frac{X_{i \Delta}-x}{h}
\end{array}\right) .
\end{aligned}
$$

When the following conditions are satisfied, the procedure terminates:

$$
\left\|\left(\begin{array}{l}
\hat{\mu}_{t}(x) \\
\hat{\mu}_{t}^{\prime}(x)
\end{array}\right)-\left(\begin{array}{c}
\hat{\mu}_{t-1}(x) \\
\hat{\mu}_{t-1}^{\prime}(x)
\end{array}\right)\right\| \leq 1 \times 10^{-4}
$$

Figure 1 shows the five sample paths of the process $X_{t}$. Table 1 lists the MSEs of the Nadaraya-Watson estimator and the variable bandwidth local M-estimator for the drift function $\mu(\cdot)$ when $n=100, n=500, n=1000, n=5000, n=10,000$. The figures in Table 1 


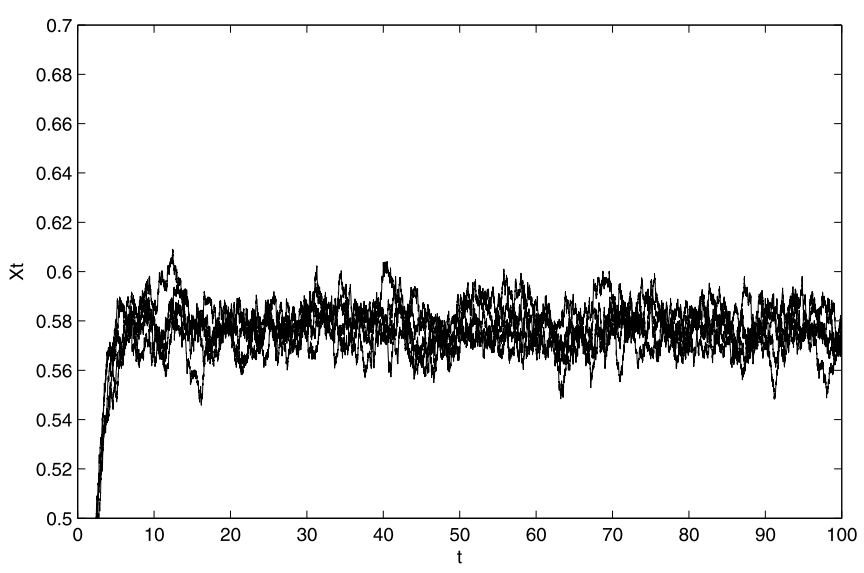

Figure 1 The five sample paths of the process $X_{t}$

Table 1 The MSEs of Nadaraya-Watson estimator $\left(\mathrm{MSE}_{1}\right)$ and variable bandwidth local M-estimator $\left(\mathrm{MSE}_{2}\right)$ for drift function $\mu(\cdot)$

\begin{tabular}{lll}
\hline Sample size $n$ & MSE $_{1}$ & MSE $_{2}$ \\
\hline$n=100$ & 0.0628 & 0.0667 \\
$n=500$ & 0.0042 & 0.0036 \\
$n=1000$ & 0.0023 & 0.0019 \\
$n=5000$ & 0.0014 & 0.0011 \\
$n=10,000$ & 0.0012 & 0.0007
\end{tabular}

indicate that

(i) The MSEs of the two types of estimators decrease toward zero as the sample size $n$ increases.

(ii) The variable bandwidth local M-estimator performs better than the Nadaraya-Watson estimator.

\section{Lemmas and proofs}

The following lemmas are needed to prove the main results of this paper.

Lemma 1 Under the conditions $\mathrm{C} 1-\mathrm{C} 5$ and the conditions (i) of the $\mathrm{C} 6-\mathrm{C} 10$, we have

$$
\begin{aligned}
& \sum_{i=1}^{n} \psi_{1}^{\prime}\left(u_{i \Delta}\right) \beta_{1}\left(X_{i \Delta}\right) K\left(\frac{X_{i \Delta}-x_{0}}{h / \beta_{1}\left(X_{i \Delta}\right)}\right)\left(X_{i \Delta}-x_{0}\right)^{l} \\
& \quad=n h^{l+1} \frac{G_{1}\left(x_{0}\right)}{\beta_{1}^{l}\left(x_{0}\right)} p\left(x_{0}\right) K_{l}\left(1+o_{p}(1)\right)
\end{aligned}
$$

and

$$
\begin{aligned}
& \sum_{i=1}^{n} \psi_{1}^{\prime}\left(u_{i \Delta}\right) R_{1}\left(X_{i \Delta}\right) \beta_{1}\left(X_{i \Delta}\right) K\left(\frac{X_{i \Delta}-x_{0}}{h / \beta_{1}\left(X_{i \Delta}\right)}\right)\left(X_{i \Delta}-x_{0}\right)^{l} \\
& =n h^{l+3} \frac{G_{1}\left(x_{0}\right)}{2 \beta_{1}^{l+2}\left(x_{0}\right)} \mu^{\prime \prime}\left(x_{0}\right) p\left(x_{0}\right) K_{l+2}\left(1+o_{p}(1)\right),
\end{aligned}
$$

where $R_{1}\left(X_{i \Delta}\right)=\mu\left(X_{i \Delta}\right)-\mu\left(x_{0}\right)-\mu^{\prime}\left(x_{0}\right)\left(X_{i \Delta}-x_{0}\right)$. 
Proof of Lemma 1 Since the second part of Lemma 1 can be proved by the same arguments as the first one, we only prove the first part. Let

$$
Z_{n, i}=\psi_{1}^{\prime}\left(u_{i \Delta}\right) \beta_{1}\left(X_{i \Delta}\right) K\left(\frac{X_{i \Delta}-x_{0}}{h / \beta_{1}\left(X_{i \Delta}\right)}\right)\left(X_{i \Delta}-x_{0}\right)^{l}
$$

By a change of variable and the continuity at the point $x_{0}$ of $\beta_{1}(\cdot), K(\cdot), G_{1}(\cdot)$ and $p(\cdot)$, we obtain

$$
\begin{aligned}
E\left(Z_{n, 1}\right) & =\int G_{1}(x) \beta_{1}(x) K\left(\frac{x-x_{0}}{h / \beta_{1}(x)}\right)\left(x-x_{0}\right)^{l} p(x) d x \\
& =\int G_{1}\left(x_{0}+y h\right) \beta_{1}\left(x_{0}+y h\right) K\left(y \beta_{1}\left(x_{0}+y h\right)\right)(y h)^{l} p\left(x_{0}+y h\right) h d y \\
& =h^{l+1} G_{1}\left(x_{0}\right) \beta_{1}\left(x_{0}\right) p\left(x_{0}\right) \int K\left(y \beta_{1}\left(x_{0}\right)\right) y^{l} d y(1+o(1)) \\
& =h^{l+1} G_{1}\left(x_{0}\right) \frac{p\left(x_{0}\right)}{\beta_{1}^{l}\left(x_{0}\right)} \int K(u) u^{l} d u(1+o(1)) \\
& =h^{l+1} G_{1}\left(x_{0}\right) \frac{p\left(x_{0}\right)}{\beta_{1}^{l}\left(x_{0}\right)} K^{l}(1+o(1)) .
\end{aligned}
$$

Therefore, we have

$$
E\left(\sum_{i=1}^{n} \psi_{1}^{\prime}\left(u_{i \Delta}\right) \beta_{1}\left(X_{i \Delta}\right) K\left(\frac{X_{i \Delta}-x_{0}}{h / \beta_{1}\left(X_{i \Delta}\right)}\right)\left(X_{i \Delta}-x_{0}\right)^{l}\right)=n h^{l+1} G_{1}\left(x_{0}\right) \frac{p\left(x_{0}\right)}{\beta_{1}^{l}\left(x_{0}\right)} K^{l}(1+o(1)) .
$$

Note that

$$
\sum_{i=1}^{n} Z_{n, i}=E\left(\sum_{i=1}^{n} Z_{n, i}\right)+O_{p}\left(\sqrt{\operatorname{Var}\left(\sum_{i=1}^{n} Z_{n, i}\right)}\right)
$$

and

$$
\operatorname{Var}\left(\sum_{i=1}^{n} Z_{n, i}\right)=n E Z_{n, 1}^{2}+2 \sum_{j=2}^{n}(n-j+1) \operatorname{Cov}\left(Z_{n, 1}, Z_{n, j}\right)
$$

By a change of variable and the continuity at the point $x_{0}$ of $\beta_{1}(\cdot), K(\cdot), G_{3}(\cdot)$ and $p(\cdot)$, we obtain

$$
\begin{aligned}
E Z_{n, 1}^{2} & =\int G_{3}(x) \beta_{1}^{2}(x) K^{2}\left(\frac{x-x_{0}}{h / \beta_{1}(x)}\right)\left(x-x_{0}\right)^{2 l} p(x) d x \\
& =\int G_{3}\left(x_{0}+y h\right) \beta_{1}^{2}\left(x_{0}+y h\right) K^{2}\left(y \beta_{1}\left(x_{0}+y h\right)\right)(y h)^{2 l} p\left(x_{0}+y h\right) h d y \\
& =h^{2 l+1} G_{3}\left(x_{0}\right) \beta_{1}^{2}\left(x_{0}\right) p\left(x_{0}\right) \int K^{2}\left(y \beta_{1}\left(x_{0}\right)\right) y^{2 l} d y(1+o(1)) \\
& =h^{2 l+1} G_{3}\left(x_{0}\right) \beta_{1}^{1-2 l}\left(x_{0}\right) p\left(x_{0}\right) \int K^{2}(u) u^{2 l} d u(1+o(1)) \\
& =O\left(h^{2 l+1}\right) .
\end{aligned}
$$


Let $d_{n}$ be a sequence of positive integers satisfying $d_{n} \rightarrow \infty$ and $h d_{n} \rightarrow 0$. Then we have

$$
\sum_{j=2}^{n}\left|\operatorname{Cov}\left(Z_{n, 1}, Z_{n, j}\right)\right|=\sum_{j=2}^{d_{n}}\left|\operatorname{Cov}\left(Z_{n, 1}, Z_{n, j}\right)\right|+\sum_{j=d_{n}+1}^{n}\left|\operatorname{Cov}\left(Z_{n, 1}, Z_{n, j}\right)\right| .
$$

By the conditions C6(i), C10(i) and the bounded support of $K(\cdot)$, we have

$$
\begin{aligned}
& \left|E Z_{n, i} Z_{n, j}\right| \\
& \leq E\left|Z_{n, i} Z_{n, j}\right| \\
& =E \mid E\left[\psi_{1}^{\prime}\left(u_{i \Delta}\right) \psi_{1}^{\prime}\left(u_{j \Delta}\right) \mid X_{i \Delta}, X_{j \Delta}\right] \beta_{1}\left(X_{i \Delta}\right) K\left(\frac{X_{i \Delta}-x_{0}}{h / \beta_{1}\left(X_{i \Delta}\right)}\right)\left(X_{i \Delta}-x_{0}\right)^{l} \\
& \quad \times \beta_{1}\left(X_{j \Delta}\right) K\left(\frac{X_{j \Delta}-x_{0}}{h / \beta_{1}\left(X_{j \Delta}\right)}\right)\left(X_{j \Delta}-x_{0}\right)^{l} \mid \\
& \leq C_{1} E\left|\beta_{1}\left(X_{i \Delta}\right) K\left(\frac{X_{i \Delta}-x_{0}}{h / \beta_{1}\left(X_{i \Delta}\right)}\right)\left(X_{i \Delta}-x_{0}\right)^{l} \beta_{1}\left(X_{j \Delta}\right) K\left(\frac{X_{j \Delta}-x_{0}}{h / \beta_{1}\left(X_{j \Delta}\right)}\right)\left(X_{j \Delta}-x_{0}\right)^{l}\right| \\
& \leq C_{2} h^{2 l+2},
\end{aligned}
$$

where $C_{1}$ and $C_{2}$ are constants. Therefore, we have

$$
\sum_{j=2}^{d_{n}}\left|\operatorname{Cov}\left(Z_{n, 1}, Z_{n, j}\right)\right| \leq C_{2} h^{2 l+2} \sum_{j=2}^{d_{n}} 1=o\left(n h^{2 l+1}\right)
$$

By using the Davydov inequality, we have

$$
\left|\operatorname{Cov}\left(Z_{n, 1}, Z_{n, j}\right)\right| \leq C_{3}[\alpha(j-1)]^{\gamma /(2+\gamma)}\left(E\left|Z_{n, 1}\right|^{2+\gamma}\right)^{2 /(2+\gamma)},
$$

and by the condition $\mathrm{C} 8(\mathrm{i})$, we have

$$
\begin{aligned}
E\left|Z_{n, i}\right|^{2+\gamma} & =E\left|E\left[\psi_{1}^{\prime}\left(u_{i \Delta}\right) \mid X_{i \Delta}\right] \beta_{1}\left(X_{i \Delta}\right) K\left(\frac{X_{i \Delta}-x_{0}}{h / \beta_{1}\left(X_{i \Delta}\right)}\right)\left(X_{i \Delta}-x_{0}\right)^{l}\right|^{2+\gamma} \\
& \leq C_{4} E\left|\beta_{1}\left(X_{i \Delta}\right) K\left(\frac{X_{i \Delta}-x_{0}}{h / \beta_{1}\left(X_{i \Delta}\right)}\right)\left(X_{i \Delta}-x_{0}\right)^{l}\right|^{2+\gamma} \\
& \leq C_{5} h^{(2+\gamma) l+1}
\end{aligned}
$$

where $C_{3}, C_{4}$ and $C_{5}$ are constants. Therefore, by using the condition $\mathrm{C} 3$ and choosing $d_{n}$ such that $d_{n}^{a} h^{\gamma /(2+\gamma)}=O(1)$, we have

$$
\begin{aligned}
\sum_{j=d_{n}+1}^{n}\left|\operatorname{Cov}\left(Z_{n, 1}, Z_{n, j}\right)\right| & \leq C_{6} \sum_{j=d_{n}+1}^{n}[\alpha(j-1)]^{\gamma / 2+\gamma}\left(h^{(2+\gamma) l+1}\right)^{2 /(2+\gamma)} \\
& =C_{6} h^{2 l+2 /(2+\gamma)} \sum_{k=d_{n}}^{n}[\alpha(k)]^{\gamma / 2+\gamma} \\
& \leq C_{6} d_{n}^{-a} h^{2 l+2 /(2+\gamma)} \sum_{k=d_{n}}^{n} k^{a}[\alpha(k)]^{\gamma / 2+\gamma}=o\left(n h^{2 l+1}\right),
\end{aligned}
$$


where $C_{6}$ is a constant. In summary, we have

$$
\operatorname{Var}\left(\sum_{i=1}^{n} Z_{n, i}\right)=O\left(n h^{2 l+1}\right)
$$

Therefore,

$$
\sum_{i=1}^{n} \psi_{1}^{\prime}\left(u_{i \Delta}\right) \beta_{1}\left(X_{i \Delta}\right) K\left(\frac{X_{i \Delta}-x_{0}}{h / \beta_{1}\left(X_{i \Delta}\right)}\right)\left(X_{i \Delta}-x_{0}\right)^{l}=n h^{l+1} \frac{G_{1}\left(x_{0}\right)}{\beta_{1}^{l}\left(x_{0}\right)} p\left(x_{0}\right) K_{l}\left(1+o_{p}(1)\right) .
$$

This completes the lemma.

Lemma 2 Under the conditions $\mathrm{C} 1-\mathrm{C} 5$ and the conditions (ii) of $\mathrm{C} 6-\mathrm{C} 10$, we have

$$
\begin{aligned}
& \sum_{i=1}^{n} \psi_{2}^{\prime}\left(v_{i \Delta}\right) \beta_{2}\left(X_{i \Delta}\right) K\left(\frac{X_{i \Delta}-x_{0}}{h / \beta_{2}\left(X_{i \Delta}\right)}\right)\left(X_{i \Delta}-x_{0}\right)^{l} \\
& \quad=n h^{l+1} \frac{H_{1}\left(x_{0}\right)}{\beta_{2}^{l}\left(x_{0}\right)} p\left(x_{0}\right) K_{l}\left(1+o_{p}(1)\right)
\end{aligned}
$$

and

$$
\begin{gathered}
\sum_{i=1}^{n} \psi_{2}^{\prime}\left(v_{i \Delta}\right) R_{2}\left(X_{i \Delta}\right) \beta_{2}\left(X_{i \Delta}\right) K\left(\frac{X_{i \Delta}-x_{0}}{h / \beta_{2}\left(X_{i \Delta}\right)}\right)\left(X_{i \Delta}-x_{0}\right)^{l} \\
=n h^{l+3} \frac{H_{1}\left(x_{0}\right)}{2 \beta_{2}^{l+2}\left(x_{0}\right)} p\left(x_{0}\right)\left(\sigma^{2}\left(x_{0}\right)\right)^{\prime \prime} K_{l+2}\left(1+o_{p}(1)\right),
\end{gathered}
$$

where $R_{2}\left(X_{i \Delta}\right)=\sigma^{2}\left(X_{i \Delta}\right)-\sigma^{2}\left(x_{0}\right)-\left(\sigma^{2}\left(x_{0}\right)\right)^{\prime}\left(X_{i \Delta}-x_{0}\right)$.

Proof of Lemma 2 Using the same techniques in proving Lemma 1, we omit the proof process.

Lemma 3 Under the conditions $\mathrm{C} 1-\mathrm{C} 5, \mathrm{C} 6-\mathrm{C} 8(\mathrm{i})$ and $\mathrm{C} 10(\mathrm{i})-13$, we have

$$
\frac{1}{\sqrt{n h}}\left(\begin{array}{c}
\sum_{i=1}^{n} \psi_{1}\left(u_{i \Delta}\right) \beta_{1}\left(X_{i \Delta}\right) K\left(\frac{X_{i \Delta}-x_{0}}{h / \beta_{1}\left(X_{i \Delta}\right)}\right) \\
\sum_{i=1}^{n} \psi_{1}\left(u_{i \Delta}\right) \beta_{1}\left(X_{i \Delta}\right) K\left(\frac{X_{i \Delta}-x_{0}}{h / \beta_{1}\left(X_{i \Delta}\right)}\right) \frac{X_{i \Delta}-x_{0}}{h}
\end{array}\right) \stackrel{D}{\rightarrow} N\left(0, \Sigma_{3}\right)
$$

where $\Sigma_{3}=G_{2}\left(x_{0}\right) p\left(x_{0}\right) \beta_{1}\left(x_{0}\right) V_{1}$.

Proof of Lemma 3 Let

$$
W_{n}=\sum_{i=1}^{n} W_{n, i}=\sum_{i=1}^{n} \psi_{1}\left(u_{i \Delta}\right) \beta_{1}\left(X_{i \Delta}\right) K\left(\frac{X_{i \Delta}-x_{0}}{h / \beta_{1}\left(X_{i \Delta}\right)}\right)\left(\begin{array}{c}
1 \\
\frac{X_{i \Delta}-x_{0}}{h}
\end{array}\right),
$$

then by the condition $C 7(\mathrm{i})$, we have $E W_{n}=0$, and

$$
\operatorname{Var} W_{n}=\operatorname{Var}\left(\sum_{i=1}^{n} W_{n, i}\right)=n E W_{n, 1}^{2}+2 \sum_{j=2}^{n}(n-j+1) \operatorname{Cov}\left(W_{n, 1}, W_{n, j}\right) .
$$


Similar to the proof methods in Lemma 1, we have

$$
\operatorname{Var} W_{n}=n h G_{2}(x) p\left(x_{0}\right) \beta_{1}\left(x_{0}\right) V_{1}(1+o(1))
$$

Next, we will show the asymptotic normality of $\frac{1}{\sqrt{n h}} W_{n}$, and this can be shown by using similar methods to Theorem 2 of [25]. This completes the lemma.

Lemma 4 Under the conditions $\mathrm{C} 1-\mathrm{C} 5, \mathrm{C} 6-\mathrm{C} 8(\mathrm{ii})$ and $\mathrm{C} 10(\mathrm{ii})-13$, we have

$$
\frac{1}{\sqrt{n h}}\left(\begin{array}{c}
\sum_{i=1}^{n} \psi_{2}\left(v_{i \Delta}\right) \beta_{2}\left(X_{i \Delta}\right) K\left(\frac{X_{i \Delta}-x_{0}}{h / \beta_{2}\left(X_{i \Delta}\right)}\right) \\
\sum_{i=1}^{n} \psi_{2}\left(v_{i \Delta}\right) \beta_{2}\left(X_{i \Delta}\right) K\left(\frac{X_{i \Delta}-x_{0}}{h / \beta_{2}\left(X_{i \Delta}\right)}\right) \frac{X_{i \Delta}-x_{0}}{h}
\end{array}\right) \stackrel{D}{\rightarrow} N\left(0, \Sigma_{4}\right)
$$

where $\Sigma_{4}=H_{2}\left(x_{0}\right) p\left(x_{0}\right) \beta_{2}\left(x_{0}\right) V_{2}$.

Proof of Lemma 4 The proof methods are similar to those used in Lemma 3.

Proof of Theorem 1 (i) We now show that the new robust estimators of $\mu(x)$ and $\mu^{\prime}(x)$ are consistent. Let

$$
\begin{aligned}
& r=\left(a_{1}, h b_{1}\right)^{T}, \quad r_{0}=\left(\mu\left(x_{0}\right), h \mu^{\prime}\left(x_{0}\right)\right)^{T}, \\
& r_{i \Delta}=\left(r-r_{0}\right)^{T}\left(\begin{array}{c}
1 \\
\frac{X_{i \Delta}-x_{0}}{h}
\end{array}\right),
\end{aligned}
$$

and

$$
L_{n}(r)=\sum_{i=1}^{n} \rho_{1}\left(\frac{X_{(i+1) \Delta}-X_{i \Delta}}{\Delta}-a_{1}-b_{1}\left(X_{i \Delta}-x_{0}\right)\right) \beta_{1}\left(X_{i \Delta}\right) K\left(\frac{X_{i \Delta}-x_{0}}{h / \beta_{1}\left(X_{i \Delta}\right)}\right) .
$$

Then we have

$$
\begin{aligned}
r_{i \Delta} & =\left(r-r_{0}\right)^{T}\left(\begin{array}{c}
1 \\
\frac{X_{i \Delta}-x_{0}}{h}
\end{array}\right) \\
& =\left(a_{1}-\mu\left(x_{0}\right), h b_{1}-h \mu^{\prime}\left(x_{0}\right)\right)\left(\begin{array}{c}
1 \\
\frac{X_{i \Delta}-x_{0}}{h}
\end{array}\right) \\
& =a_{1}-\mu\left(x_{0}\right)+\left(h b_{1}-h \mu^{\prime}\left(x_{0}\right)\right) \frac{X_{i \Delta}-x_{0}}{h} \\
& =a_{1}-\mu\left(x_{0}\right)+\left(b_{1}-\mu^{\prime}\left(x_{0}\right)\right)\left(X_{i \Delta}-x_{0}\right) \\
& =a_{1}+b_{1}\left(X_{i \Delta}-x_{0}\right)-\mu\left(x_{0}\right)-\mu^{\prime}\left(x_{0}\right)\left(X_{i \Delta}-x_{0}\right) \\
& =a_{1}+b_{1}\left(X_{i \Delta}-x_{0}\right)+R_{1}\left(X_{i \Delta}\right)-\mu\left(X_{i \Delta}\right) \\
& =a_{1}+b_{1}\left(X_{i \Delta}-x_{0}\right)+R_{1}\left(X_{i \Delta}\right)-\left(\frac{X_{(i+1) \Delta}-X_{i \Delta}}{\Delta}-u_{i \Delta}\right) .
\end{aligned}
$$

$\mathrm{W}$ denote the circle centered at $r_{0}$ and with radius $\delta$ by $S_{\delta} . \forall \delta>0$, we prove

$$
\lim _{n \rightarrow \infty} P\left\{\inf _{r \in S_{\delta}} L_{n}(r)>L_{n}\left(r_{0}\right)\right\}=1
$$


For $r \in S_{\delta}$,

$$
\begin{aligned}
& L_{n}(r)-L_{n}\left(r_{0}\right) \\
& =\sum_{i=1}^{n} \rho_{1}\left(\frac{X_{(i+1) \Delta}-X_{i \Delta}}{\Delta}-a_{1}-b_{1}\left(X_{i \Delta}-x_{0}\right)\right) \beta_{1}\left(X_{i \Delta}\right) K\left(\frac{X_{i \Delta}-x_{0}}{h / \beta_{1}\left(X_{i \Delta}\right)}\right) \\
& -\sum_{i=1}^{n} \rho_{1}\left(\frac{X_{(i+1) \Delta}-X_{i \Delta}}{\Delta}-\mu\left(x_{0}\right)-\mu^{\prime}\left(x_{0}\right)\left(X_{i \Delta}-x_{0}\right)\right) \beta_{1}\left(X_{i \Delta}\right) K\left(\frac{X_{i \Delta}-x_{0}}{h / \beta_{1}\left(X_{i \Delta}\right)}\right) \\
& =\sum_{i=1}^{n} \beta_{1}\left(X_{i \Delta}\right) K\left(\frac{X_{i \Delta}-x_{0}}{h / \beta_{1}\left(X_{i \Delta}\right)}\right)\left[\rho_{1}\left(u_{i \Delta}+R_{1}\left(X_{i \Delta}\right)-r_{i \Delta}\right)-\rho_{1}\left(u_{i \Delta}+R_{1}\left(X_{i \Delta}\right)\right)\right] \\
& =\sum_{i=1}^{n} \beta_{1}\left(X_{i \Delta}\right) K\left(\frac{X_{i \Delta}-x_{0}}{h / \beta_{1}\left(X_{i \Delta}\right)}\right) \int_{u_{i \Delta}+R_{1}\left(X_{i \Delta}\right)}^{u_{i \Delta}+R_{1}\left(X_{i \Delta}\right)-r_{i \Delta}} \psi_{1}(t) d t \\
& =\sum_{i=1}^{n} \beta_{1}\left(X_{i \Delta}\right) K\left(\frac{X_{i \Delta}-x_{0}}{h / \beta_{1}\left(X_{i \Delta}\right)}\right) \int_{u_{i \Delta}+R_{1}\left(X_{i \Delta}\right)}^{u_{i \Delta}+R_{1}\left(X_{i \Delta}\right)-r_{i \Delta}} \psi_{1}\left(u_{i \Delta}\right) d t \\
& +\sum_{i=1}^{n} \beta_{1}\left(X_{i \Delta}\right) K\left(\frac{X_{i \Delta}-x_{0}}{h / \beta_{1}\left(X_{i \Delta}\right)}\right) \int_{u_{i \Delta}+R_{1}\left(X_{i \Delta}\right)}^{u_{i \Delta}+R_{1}\left(X_{i \Delta}\right)-r_{i \Delta}} \psi_{1}^{\prime}\left(u_{i \Delta}\right)\left(t-u_{i \Delta}\right) d t \\
& +\sum_{i=1}^{n} \beta_{1}\left(X_{i \Delta}\right) K\left(\frac{X_{i \Delta}-x_{0}}{h / \beta_{1}\left(X_{i \Delta}\right)}\right) \\
& \times \int_{u_{i \Delta}+R_{1}\left(X_{i \Delta}\right)}^{u_{i \Delta}+R_{1}\left(X_{i \Delta}\right)-r_{i \Delta}}\left[\psi_{1}(t)-\psi_{1}\left(u_{i \Delta}\right)-\psi_{1}^{\prime}\left(u_{i \Delta}\right)\left(t-u_{i \Delta}\right)\right] d t \\
& =: L_{n 1}+L_{n 2}+L_{n 3} \text {. }
\end{aligned}
$$

Next, we will show that

$$
\begin{aligned}
& L_{n 1}=o_{p}(n h \delta), \\
& L_{n 2}=\frac{n h}{2}\left(r-r_{0}\right)^{T} G_{1}\left(x_{0}\right) p\left(x_{0}\right) U_{1}\left(1+o_{p}(1)\right)\left(r-r_{0}\right)+O_{p}\left(n h^{3} \delta\right), \\
& L_{n 3}=o_{p}\left(n h \delta^{2}\right) .
\end{aligned}
$$

For (7), we have

$$
\begin{aligned}
L_{n 1} & =\sum_{i=1}^{n} \beta_{1}\left(X_{i \Delta}\right) K\left(\frac{X_{i \Delta}-x_{0}}{h / \beta_{1}\left(X_{i \Delta}\right)}\right) \int_{u_{i \Delta}+R_{1}\left(X_{i \Delta}\right)}^{u_{i \Delta}+R_{1}\left(X_{i \Delta}\right)-r_{i \Delta}} \psi_{1}\left(u_{i \Delta}\right) d t \\
& =\sum_{i=1}^{n} \beta_{1}\left(X_{i \Delta}\right) K\left(\frac{X_{i \Delta}-x_{0}}{h / \beta_{1}\left(X_{i \Delta}\right)}\right) \psi_{1}\left(u_{i \Delta}\right)\left(-r_{i \Delta}\right) \\
& =-\left(r-r_{0}\right)^{T} \sum_{i=1}^{n} \beta_{1}\left(X_{i \Delta}\right) K\left(\frac{X_{i \Delta}-x_{0}}{h / \beta_{1}\left(X_{i \Delta}\right)}\right) \psi_{1}\left(u_{i \Delta}\right)\left(\begin{array}{c}
1 \\
\frac{X_{i \Delta}-x_{0}}{h}
\end{array}\right) \\
& =-\left(r-r_{0}\right)^{T} W_{n},
\end{aligned}
$$


where

$$
W_{n}=\sum_{i=1}^{n} W_{n, i}=\sum_{i=1}^{n} \psi_{1}\left(u_{i \Delta}\right) \beta_{1}\left(X_{i \Delta}\right) K\left(\frac{X_{i \Delta}-x_{0}}{h / \beta_{1}\left(X_{i \Delta}\right)}\right)\left(\begin{array}{c}
1 \\
\frac{X_{i \Delta}-x_{0}}{h}
\end{array}\right) .
$$

By the proof of Lemma 3, we have $E W_{n}=0$, and

$\operatorname{Var} W_{n}=n h G_{2}(x) p\left(x_{0}\right) \beta_{1}\left(x_{0}\right) V_{1}(1+o(1))$.

Note that

$$
W_{n}=\sum_{i=1}^{n} W_{n, i}=E\left(\sum_{i=1}^{n} W_{n, i}\right)+O_{p}\left(\sqrt{\operatorname{Var}\left(\sum_{i=1}^{n} W_{n, i}\right)}\right),
$$

so we have $W_{n}=O_{p}(\sqrt{n h})$, which means that (7) holds.

For (8), we have

$$
\begin{aligned}
L_{n 2}= & \sum_{i=1}^{n} \beta_{1}\left(X_{i \Delta}\right) K\left(\frac{X_{i \Delta}-x_{0}}{h / \beta_{1}\left(X_{i \Delta}\right)}\right) \int_{u_{i \Delta}+R_{1}\left(X_{i \Delta}\right)}^{u_{i \Delta}+R_{1}\left(X_{i \Delta}\right)-r_{i \Delta}}\left[\psi_{1}^{\prime}\left(u_{i \Delta}\right)\left(t-u_{i \Delta}\right)\right] d t \\
= & \frac{1}{2} \sum_{i=1}^{n} \beta_{1}\left(X_{i \Delta}\right) K\left(\frac{X_{i \Delta}-x_{0}}{h / \beta_{1}\left(X_{i \Delta}\right)}\right) \psi_{1}^{\prime}\left(u_{i \Delta}\right)\left(r_{i \Delta}^{2}-2 R_{1}\left(X_{i \Delta}\right) r_{i \Delta}\right) \\
= & \frac{1}{2} \sum_{i=1}^{n} \beta_{1}\left(X_{i \Delta}\right) K\left(\frac{X_{i \Delta}-x_{0}}{h / \beta_{1}\left(X_{i \Delta}\right)}\right) \psi_{1}^{\prime}\left(u_{i \Delta}\right)\left(r-r_{0}\right)^{T}\left(\begin{array}{cc}
1 & \frac{X_{i \Delta}-x_{0}}{h} \\
\frac{X_{i \Delta}-x_{0}}{h} & \frac{\left(X_{i \Delta}-x_{0}\right)^{2}}{h^{2}}
\end{array}\right)\left(r-r_{0}\right) \\
& -\sum_{i=1}^{n} \beta_{1}\left(X_{i \Delta}\right) K\left(\frac{X_{i \Delta}-x_{0}}{h / \beta_{1}\left(X_{i \Delta}\right)}\right) \psi_{1}^{\prime}\left(u_{i \Delta}\right) R_{1}\left(X_{i \Delta}\right) r_{i \Delta} \\
= & L_{n 21}+L_{n 22} .
\end{aligned}
$$

From Lemma 1 with $l=0, l=1$ and $l=2$, we get

$$
\begin{aligned}
L_{n 21} & =\frac{1}{2} \sum_{i=1}^{n} \beta_{1}\left(X_{i \Delta}\right) K\left(\frac{X_{i \Delta}-x_{0}}{h / \beta_{1}\left(X_{i \Delta}\right)}\right) \psi_{1}^{\prime}\left(u_{i \Delta}\right)\left(r-r_{0}\right)^{T}\left(\begin{array}{cc}
1 & \frac{X_{i \Delta}-x_{0}}{h} \\
\frac{X_{i \Delta}-x_{0}}{h} & \frac{\left(X_{i \Delta}-x_{0}\right)^{2}}{h^{2}}
\end{array}\right)\left(r-r_{0}\right) \\
& =\frac{n h}{2}\left(r-r_{0}\right)^{T} G_{1}\left(x_{0}\right) p\left(x_{0}\right)\left(\begin{array}{cc}
K_{0} & \frac{K_{1}}{\beta_{1}\left(x_{0}\right)} \\
\frac{K_{1}}{\beta_{1}\left(x_{0}\right)} & \frac{K_{2}}{\beta_{1}^{2}\left(x_{0}\right)}
\end{array}\right)\left(1+o_{p}(1)\right)\left(r-r_{0}\right) \\
& =\frac{n h}{2}\left(r-r_{0}\right)^{T} G_{1}\left(x_{0}\right) p\left(x_{0}\right) U_{1}\left(1+o_{p}(1)\right)\left(r-r_{0}\right)
\end{aligned}
$$

and

$$
\begin{aligned}
L_{n 22} & =-\sum_{i=1}^{n} \beta_{1}\left(X_{i \Delta}\right) K\left(\frac{X_{i \Delta}-x_{0}}{h / \beta_{1}\left(X_{i \Delta}\right)}\right) \psi_{1}^{\prime}\left(u_{i \Delta}\right) R_{1}\left(X_{i \Delta}\right) r_{i \Delta} \\
& =-\left(r-r_{0}\right)^{T} \sum_{i=1}^{n} \beta_{1}\left(X_{i \Delta}\right) K\left(\frac{X_{i \Delta}-x_{0}}{h / \beta_{1}\left(X_{i \Delta}\right)}\right) \psi_{1}^{\prime}\left(u_{i \Delta}\right) R_{1}\left(X_{i \Delta}\right)\left(\begin{array}{c}
1 \\
\frac{X_{i \Delta}-x_{0}}{h}
\end{array}\right)
\end{aligned}
$$




$$
\begin{aligned}
& =-\frac{n h^{3}}{2}\left(r-r_{0}\right)^{T} G_{1}\left(x_{0}\right) \mu^{\prime \prime}\left(x_{0}\right) p\left(x_{0}\right)\left(\begin{array}{c}
\frac{K_{2}}{\beta_{1}^{2}\left(x_{0}\right)} \\
\frac{K_{3}}{\beta_{1}^{3}\left(x_{0}\right)}
\end{array}\right)\left(1+o_{p}(1)\right) \\
& =O_{p}\left(n h^{3} \delta\right) .
\end{aligned}
$$

Therefore,

$$
\begin{aligned}
L_{n 2} & =L_{n 21}+L_{n 22} \\
& =\frac{n h}{2}\left(r-r_{0}\right)^{T} G_{1}\left(x_{0}\right) p\left(x_{0}\right) U_{1}\left(1+o_{p}(1)\right)\left(r-r_{0}\right)+O_{p}\left(n h^{3} \delta\right) .
\end{aligned}
$$

For (9), by the integral mean value theorem, we have

$$
\begin{aligned}
L_{n 3}= & \sum_{i=1}^{n} \beta_{1}\left(X_{i \Delta}\right) K\left(\frac{X_{i \Delta}-x_{0}}{h / \beta_{1}\left(X_{i \Delta}\right)}\right) \\
& \times \int_{u_{i \Delta}+R_{1}\left(X_{i \Delta}\right)}^{u_{i \Delta}+R_{1}\left(X_{i \Delta}\right)-r_{i \Delta}}\left[\psi_{1}(t)-\psi_{1}\left(u_{i \Delta}\right)-\psi_{1}^{\prime}\left(u_{i \Delta}\right)\left(t-u_{i \Delta}\right)\right] d t \\
= & \sum_{i=1}^{n} \beta_{1}\left(X_{i \Delta}\right) K\left(\frac{X_{i \Delta}-x_{0}}{h / \beta_{1}\left(X_{i \Delta}\right)}\right) \int_{R_{1}\left(X_{i \Delta}\right)}^{R_{1}\left(X_{i \Delta}\right)-r_{i \Delta}}\left[\psi_{1}\left(t+u_{i \Delta}\right)-\psi_{1}\left(u_{i \Delta}\right)-\psi_{1}^{\prime}\left(u_{i \Delta}\right) t\right] d t \\
= & \sum_{i=1}^{n} \beta_{1}\left(X_{i \Delta}\right) K\left(\frac{X_{i \Delta}-x_{0}}{h / \beta_{1}\left(X_{i \Delta}\right)}\right)\left[\psi_{1}\left(z_{i \Delta}+u_{i \Delta}\right)-\psi_{1}\left(u_{i \Delta}\right)-\psi_{1}^{\prime}\left(u_{i \Delta}\right) z_{i \Delta}\right]\left(-r_{i \Delta}\right) \\
= & -\left(r-r_{0}\right)^{T} \sum_{i=1}^{n} \beta_{1}\left(X_{i \Delta}\right) K\left(\frac{X_{i \Delta}-x_{0}}{h / \beta_{1}\left(X_{i \Delta}\right)}\right)\left[\psi_{1}\left(z_{i \Delta}+u_{i \Delta}\right)-\psi_{1}\left(u_{i \Delta}\right)-\psi_{1}^{\prime}\left(u_{i \Delta}\right) z_{i \Delta}\right] \\
& \times\left(\begin{array}{c}
1 \\
\frac{X_{i \Delta}-x_{0}}{h}
\end{array}\right),
\end{aligned}
$$

where $z_{i \Delta}(i=1,2, \ldots, n)$ lies between $R_{1}\left(X_{i \Delta}\right)$ and $R_{1}\left(X_{i \Delta}\right)-r_{i \Delta}$.

By $\left|X_{i \Delta}-x_{0}\right| \leq \frac{h}{\min _{x} \beta_{1}(x)}$, we have

$$
\begin{aligned}
\max _{i}\left|z_{i \Delta}\right| & \leq \max _{i}\left|R_{1}\left(X_{i \Delta}\right)\right|+\left|\left(r-r_{0}\right)^{T}\left(\begin{array}{c}
1 \\
\frac{X_{i \Delta}-x_{0}}{h}
\end{array}\right)\right| \\
& \leq \max _{i}\left|R_{1}\left(X_{i \Delta}\right)\right|+\left(1+\frac{1}{\min _{x} \beta_{1}(x)}\right) \delta,
\end{aligned}
$$

and according to Taylor's expansion,

$$
\begin{aligned}
\max _{i}\left|R_{1}\left(X_{i \Delta}\right)\right| & =\max _{i}\left|\mu\left(X_{i \Delta}\right)-\mu\left(x_{0}\right)-\mu^{\prime}\left(x_{0}\right)\left(X_{i \Delta}-x_{0}\right)\right| \\
& =\max _{i}\left|\frac{1}{2} \mu^{\prime \prime}\left(\xi_{i}\right)\left(X_{i \Delta}-x_{0}\right)^{2}\right| \\
& \leq O_{p}\left(h^{2}\right),
\end{aligned}
$$

where $\xi_{i}$ lies between $x_{0}$ and $X_{i \Delta}, i=1,2, \ldots, n$. 
$\forall \eta>0$, let $D_{\eta}=\left\{\left(\delta_{1 \Delta}, \delta_{2 \Delta}, \ldots, \delta_{n \Delta}\right)^{T}:\left|\delta_{i \Delta}\right| \leq \eta, \forall i \leq n\right\}$, by the condition C9(i) and $\left|X_{i \Delta}-x_{0}\right| \leq \frac{h}{\min _{x} \beta_{1}(x)}$, we get

$$
\begin{aligned}
& E\left[\sup _{D_{\eta}}\left|\sum_{i=1}^{n}\left[\psi_{1}\left(\delta_{i \Delta}+u_{i \Delta}\right)-\psi_{1}\left(u_{i \Delta}\right)-\psi_{1}^{\prime}\left(u_{i \Delta}\right) \delta_{i \Delta}\right] \beta_{1}\left(X_{i \Delta}\right) K\left(\frac{X_{i \Delta}-x_{0}}{h / \beta_{1}\left(X_{i \Delta}\right)}\right)\left(X_{i \Delta}-x_{0}\right)^{l}\right|\right] \\
& \leq E\left[\sum_{i=1}^{n} \sup _{D_{\eta}}\left|\psi_{1}\left(\delta_{i \Delta}+u_{i \Delta}\right)-\psi_{1}\left(u_{i \Delta}\right)-\psi_{1}^{\prime}\left(u_{i \Delta}\right) \delta_{i \Delta}\right| \beta_{1}\left(X_{i \Delta}\right)\right. \\
& \left.\quad \times K\left(\frac{X_{i \Delta}-x_{0}}{h / \beta_{1}\left(X_{i \Delta}\right)}\right)\left|X_{i \Delta}-x_{0}\right|^{l}\right] \\
& \leq a_{\eta} \delta E\left[\sum_{i=1}^{n} \beta_{1}\left(X_{i \Delta}\right) K\left(\frac{X_{i \Delta}-x_{0}}{h / \beta_{1}\left(X_{i \Delta}\right)}\right)\left|X_{i \Delta}-x_{0}\right|^{l}\right] \\
& \leq b_{\eta} \delta
\end{aligned}
$$

where $a_{\eta}>0, b_{\eta}>0$ are two sequences, and satisfy $a_{\eta} \rightarrow 0$ and $b_{\eta} \rightarrow 0$ as $\eta \rightarrow 0$. From (10) and (11), we can see that

$$
\begin{aligned}
& \sum_{i=1}^{n}\left[\psi_{1}\left(z_{i \Delta}+u_{i \Delta}\right)-\psi_{1}\left(u_{i \Delta}\right)-\psi_{1}^{\prime}\left(u_{i \Delta}\right) z_{i \Delta}\right] \beta_{1}\left(X_{i \Delta}\right) K\left(\frac{X_{i \Delta}-x_{0}}{h / \beta_{1}\left(X_{i \Delta}\right)}\right)\left(X_{i \Delta}-x_{0}\right)^{l} \\
& \quad=o_{p}\left(n h^{l+1} \delta\right),
\end{aligned}
$$

we get (9) immediately.

Let $U_{1}$ be a positive definite matrix, $\lambda$ be the smallest eigenvalue of the $U_{1}$. Accordingly, for any $r \in S_{\delta}$,

$$
\begin{aligned}
& L_{n}(r)-L_{n}\left(r_{0}\right) \\
& \quad=L_{n 1}+L_{n 2}+L_{n 3} \\
& \quad=\frac{n h}{2} G_{1}\left(x_{0}\right) p\left(x_{0}\right)\left(r-r_{0}\right)^{T} U_{1}\left(r-r_{0}\right)\left(1+o_{p}(1)\right)+O_{p}\left(n h^{3} \delta\right) \\
& \quad \geq \frac{n h}{2} G_{1}\left(x_{0}\right) p\left(x_{0}\right) \lambda \delta^{2}\left(1+o_{p}(1)\right)+O_{p}\left(n h^{3} \delta\right) .
\end{aligned}
$$

So as $n \rightarrow \infty$, we have

$$
P\left\{\inf _{r \in S_{\delta}} L_{n}(r)-L_{n}\left(r_{0}\right)>\frac{n h}{2} G_{1}\left(x_{0}\right) p\left(x_{0}\right) \lambda \delta^{2}>0\right\} \rightarrow 1
$$

it follows that (6) holds. In view of (6), one can easily see that $L_{n}(r)$ has a local minimum in the interior of $S_{\delta}$, thus there exist solutions to equation (4). Let $\left(h \hat{\mu}\left(x_{0}\right), h \hat{\mu}^{\prime}\left(x_{0}\right)\right)^{T}$ denote the closest solution to $r_{0}=\left(\mu\left(x_{0}\right), \mu^{\prime}\left(x_{0}\right)\right)^{T}$, then

$$
\lim _{n \rightarrow \infty} P\left\{\left(\hat{\mu}\left(x_{0}\right)-\mu\left(x_{0}\right)\right)^{2}+h^{2}\left(\hat{\mu}^{\prime}\left(x_{0}\right)-\mu^{\prime}\left(x_{0}\right)\right)^{2} \leq \delta^{2}\right\}=1
$$

we finish the proof of the consistency of the proposed estimators of $\mu(x)$ and $\mu^{\prime}(x)$. 
(ii) We derive the asymptotic normality of the new robust estimators of $\mu(x)$ and $\mu^{\prime}(x)$. Let

$$
\hat{\eta}_{i \Delta}=R_{1}\left(X_{i \Delta}\right)-\left(\hat{\mu}\left(x_{0}\right)-\mu\left(x_{0}\right)\right)-\left(\hat{\mu}^{\prime}\left(x_{0}\right)-\mu^{\prime}\left(x_{0}\right)\right)\left(X_{i \Delta}-x_{0}\right) .
$$

Then we have

$$
\begin{aligned}
\frac{X_{(i+1) \Delta}-X_{i \Delta}}{\Delta} & =\mu\left(X_{i \Delta}\right)+u_{i \Delta} \\
& =u_{i \Delta}+\mu\left(X_{i \Delta}\right)-\mu\left(x_{0}\right)-\mu^{\prime}\left(x_{0}\right)\left(X_{i \Delta}-x_{0}\right)+\mu\left(x_{0}\right)+\mu^{\prime}\left(x_{0}\right)\left(X_{i \Delta}-x_{0}\right) \\
& =u_{i \Delta}+R_{1}\left(X_{i \Delta}\right)+\hat{\mu}\left(x_{0}\right)+\hat{\mu}^{\prime}\left(x_{0}\right)\left(X_{i \Delta}-x_{0}\right)+\hat{\eta}_{i \Delta}-R_{1}\left(X_{i \Delta}\right) \\
& =\hat{\mu}\left(x_{0}\right)+\hat{\mu}^{\prime}\left(x_{0}\right)\left(X_{i \Delta}-x_{0}\right)+u_{i \Delta}+\hat{\eta}_{i \Delta} .
\end{aligned}
$$

Therefore by (4), we have

$$
\sum_{i=1}^{n} \psi_{1}\left(u_{i \Delta}+\hat{\eta}_{i \Delta}\right) \beta_{1}\left(X_{i \Delta}\right) K\left(\frac{X_{i \Delta}-x}{h / \beta_{1}\left(X_{i \Delta}\right)}\right)\left(\begin{array}{c}
1 \\
\frac{X_{i \Delta}-x}{h}
\end{array}\right)=0 .
$$

Let

$$
\begin{aligned}
T_{n 1} & =\sum_{i=1}^{n} \psi_{1}\left(u_{i \Delta}\right) \beta_{1}\left(X_{i \Delta}\right) K\left(\frac{X_{i \Delta}-x}{h / \beta_{1}\left(X_{i \Delta}\right)}\right)\left(\begin{array}{c}
1 \\
\frac{X_{i \Delta}-x}{h}
\end{array}\right)=W_{n}, \\
T_{n 2} & =\sum_{i=1}^{n} \psi_{1}^{\prime}\left(u_{i \Delta}\right) \hat{\eta}_{i \Delta} \beta_{1}\left(X_{i \Delta}\right) K\left(\frac{X_{i \Delta}-x}{h / \beta_{1}\left(X_{i \Delta}\right)}\right)\left(\begin{array}{c}
1 \\
\frac{X_{i \Delta}-x}{h}
\end{array}\right), \\
T_{n 3} & =\sum_{i=1}^{n}\left[\psi_{1}\left(u_{i \Delta}+\hat{\eta}_{i \Delta}\right)-\psi_{1}\left(u_{i \Delta}\right)-\psi_{1}^{\prime}\left(u_{i \Delta}\right) \hat{\eta}_{i \Delta}\right] \beta_{1}\left(X_{i \Delta}\right) K\left(\frac{X_{i \Delta}-x}{h / \beta_{1}\left(X_{i \Delta}\right)}\right)\left(\begin{array}{c}
1 \\
\frac{X_{i \Delta}-x}{h}
\end{array}\right) .
\end{aligned}
$$

In view of (13), one can get $T_{n 1}+T_{n 2}+T_{n 3}=0$. Lemma 1 and (12) imply that

$$
\begin{aligned}
T_{n 2}= & \sum_{i=1}^{n} \psi_{1}^{\prime}\left(u_{i \Delta}\right) R_{1}\left(X_{i \Delta}\right) \beta_{1}\left(X_{i \Delta}\right) K\left(\frac{X_{i \Delta}-x}{h / \beta_{1}\left(X_{i \Delta}\right)}\right)\left(\begin{array}{c}
1 \\
\frac{X_{i \Delta}-x}{h}
\end{array}\right)-\sum_{i=1}^{n} \psi_{1}^{\prime}\left(u_{i \Delta}\right) \beta_{1}\left(X_{i \Delta}\right) \\
& \cdot K\left(\frac{X_{i \Delta}-x}{h / \beta_{1}\left(X_{i \Delta}\right)}\right)\left(\begin{array}{c}
\left(\hat{\mu}\left(x_{0}\right)-\mu\left(x_{0}\right)\right)+\left(\hat{\mu}^{\prime}\left(x_{0}\right)-\mu^{\prime}\left(x_{0}\right)\right)\left(X_{i \Delta}-x_{0}\right) \\
\frac{X_{i \Delta}-x}{h}\left[\left(\hat{\mu}\left(x_{0}\right)-\mu\left(x_{0}\right)\right)+\left(\hat{\mu}^{\prime}\left(x_{0}\right)-\mu^{\prime}\left(x_{0}\right)\right)\left(X_{i \Delta}-x_{0}\right)\right]
\end{array}\right) \\
= & \sum_{i=1}^{n} \psi_{1}^{\prime}\left(u_{i \Delta}\right) R_{1}\left(X_{i \Delta}\right) \beta_{1}\left(X_{i \Delta}\right) K\left(\frac{X_{i \Delta}-x}{h / \beta_{1}\left(X_{i \Delta}\right)}\right)\left(\begin{array}{c}
1 \\
\frac{X_{i \Delta}-x}{h}
\end{array}\right) \\
& -\sum_{i=1}^{n} \psi_{1}^{\prime}\left(u_{i \Delta}\right) \beta_{1}\left(X_{i \Delta}\right) K\left(\frac{X_{i \Delta}-x}{h / \beta_{1}\left(X_{i \Delta}\right)}\right)\left(\begin{array}{cc}
1 & \frac{X_{i \Delta}-x}{h} \\
\frac{X_{i \Delta}-x}{h} & \frac{\left(X_{i \Delta}-x\right)^{2}}{h^{2}}
\end{array}\right)\left(\begin{array}{c}
\hat{\mu}\left(x_{0}\right)-\mu\left(x_{0}\right) \\
h\left(\hat{\mu}^{\prime}\left(x_{0}\right)-\mu^{\prime}\left(x_{0}\right)\right)
\end{array}\right) \\
= & \frac{n h^{3}}{2} G_{1}\left(x_{0}\right) \mu^{\prime \prime}\left(x_{0}\right) p\left(x_{0}\right)\left(\begin{array}{c}
\frac{K_{2}}{\beta_{1}^{2}\left(x_{0}\right)} \\
\frac{K_{3}}{\beta_{1}^{3}\left(x_{0}\right)}
\end{array}\right)\left(1+o_{p}(1)\right) \\
& -n h G_{1}\left(x_{0}\right) p\left(x_{0}\right)\left(\begin{array}{cc}
K_{0} & \frac{K_{1}}{\beta_{1}\left(x_{0}\right)} \\
\frac{K_{1}}{\beta_{1}\left(x_{0}\right)} & \frac{K_{2}^{2}}{\beta_{1}^{2}\left(x_{0}\right)}
\end{array}\right)\left(1+o_{p}(1)\right)\left(\begin{array}{c}
\hat{\mu}\left(x_{0}\right)-\mu\left(x_{0}\right) \\
h\left(\hat{\mu}^{\prime}\left(x_{0}\right)-\mu^{\prime}\left(x_{0}\right)\right)
\end{array}\right)
\end{aligned}
$$




$$
\begin{aligned}
= & \frac{n h^{3} G_{1}\left(x_{0}\right) \mu^{\prime \prime}\left(x_{0}\right) p\left(x_{0}\right)}{2 \beta_{1}^{2}\left(x_{0}\right)} A_{1}\left(1+o_{p}(1)\right) \\
& -n h G_{1}\left(x_{0}\right) p\left(x_{0}\right) U_{1}\left(1+o_{p}(1)\right)\left(\begin{array}{c}
\hat{\mu}\left(x_{0}\right)-\mu\left(x_{0}\right) \\
h\left(\hat{\mu}^{\prime}\left(x_{0}\right)-\mu^{\prime}\left(x_{0}\right)\right)
\end{array}\right)=: T_{n 21}+T_{n 22} .
\end{aligned}
$$

Since we have already got the consistency of $\left(\hat{\mu}\left(x_{0}\right), h \hat{\mu}^{\prime}\left(x_{0}\right)\right)$ and using $\left|X_{i \Delta}-x_{0}\right| \leq$ $\frac{h}{\min _{x} \beta_{1}(x)}$, we have

$$
\begin{aligned}
\sup _{i}\left|\hat{\eta}_{i \Delta}\right| & =\sup _{i}\left|R_{1}\left(X_{i \Delta}\right)-\left(\hat{\mu}\left(x_{0}\right)-\mu\left(x_{0}\right)\right)-\left(\hat{\mu}^{\prime}\left(x_{0}\right)-\mu^{\prime}\left(x_{0}\right)\right)\left(X_{i \Delta}-x_{0}\right)\right| \\
& \leq \sup _{i}\left|R_{1}\left(X_{i \Delta}\right)\right|+\left|\hat{\mu}\left(x_{0}\right)-\mu\left(x_{0}\right)\right|+\frac{h}{\min _{x} \beta_{1}(x)}\left|\hat{\mu}^{\prime}\left(x_{0}\right)-\mu^{\prime}\left(x_{0}\right)\right| \\
& =O_{p}\left(h^{2}+\left(\hat{\mu}\left(x_{0}\right)-\mu\left(x_{0}\right)\right)+h\left(\hat{\mu}^{\prime}\left(x_{0}\right)-\mu^{\prime}\left(x_{0}\right)\right)\right)=o_{p}(1),
\end{aligned}
$$

then, by the condition C9(i) and the same argument as that in the first part of Theorem 1, we have

$$
\begin{aligned}
T_{n 3} & =\sum_{i=1}^{n}\left[\psi_{1}\left(u_{i \Delta}+\hat{\eta}_{i \Delta}\right)-\psi_{1}\left(u_{i \Delta}\right)-\psi_{1}^{\prime}\left(u_{i \Delta}\right) \hat{\eta}_{i \Delta}\right] \beta_{1}\left(X_{i \Delta}\right) K\left(\frac{X_{i \Delta}-x}{h / \beta_{1}\left(X_{i \Delta}\right)}\right)\left(\begin{array}{c}
1 \\
\frac{X_{i \Delta}-x}{h}
\end{array}\right) \\
& =o_{p}(n h)\left[h^{2}+\left(\hat{\mu}\left(x_{0}\right)-\mu\left(x_{0}\right)\right)+h\left(\hat{\mu}^{\prime}\left(x_{0}\right)-\mu^{\prime}\left(x_{0}\right)\right)\right]=o_{p}\left(T_{n 22}\right) .
\end{aligned}
$$

Therefore, using the fact that $T_{n 1}+T_{n 2}+T_{n 3}=0$, we have

$$
\begin{aligned}
& \left(\begin{array}{c}
\hat{\mu}\left(x_{0}\right)-\mu\left(x_{0}\right) \\
h\left(\hat{\mu}^{\prime}\left(x_{0}\right)-\mu^{\prime}\left(x_{0}\right)\right)
\end{array}\right) \\
& \quad=\frac{1}{n h} G_{1}^{-1}\left(x_{0}\right) p^{-1}\left(x_{0}\right) U_{1}^{-1}\left(1+o_{p}(1)\right) W_{n}+\frac{h^{2}}{2 \beta_{1}^{2}\left(x_{0}\right)} \mu^{\prime \prime}\left(x_{0}\right) U_{1}^{-1} A_{1}\left(1+o_{p}(1)\right)
\end{aligned}
$$

it follows that

$$
\begin{aligned}
& \sqrt{n h}\left[\left(\begin{array}{c}
\hat{\mu}\left(x_{0}\right)-\mu\left(x_{0}\right) \\
h\left(\hat{\mu}^{\prime}\left(x_{0}\right)-\mu^{\prime}\left(x_{0}\right)\right)
\end{array}\right)-\frac{h^{2} \mu^{\prime \prime}\left(x_{0}\right)}{2 \beta_{1}^{2}\left(x_{0}\right)} U_{1}^{-1} A_{1}\left(1+o_{p}(1)\right)\right] \\
& =G_{1}^{-1}\left(x_{0}\right) p^{-1}\left(x_{0}\right) U_{1}^{-1}\left(1+o_{p}(1)\right) \frac{1}{\sqrt{n h}} W_{n} .
\end{aligned}
$$

According to Lemma 3 and the Slutsky theorem, we have

$$
\begin{aligned}
& \sqrt{n h}\left[\left(\begin{array}{c}
\hat{\mu}\left(x_{0}\right)-\mu\left(x_{0}\right) \\
h\left(\hat{\mu}^{\prime}\left(x_{0}\right)-\mu^{\prime}\left(x_{0}\right)\right)
\end{array}\right)-\frac{h^{2} \mu^{\prime \prime}\left(x_{0}\right)}{2 \beta_{1}^{2}\left(x_{0}\right)} U_{1}^{-1} A_{1}\right] \\
& \stackrel{D}{\rightarrow} G_{1}^{-1}\left(x_{0}\right) p^{-1}\left(x_{0}\right) U_{1}^{-1} N\left(0, \Sigma_{3}\right) \\
& =N\left(0, \frac{G_{2}\left(x_{0}\right) \beta_{1}\left(x_{0}\right)}{G_{1}^{2}\left(x_{0}\right) p\left(x_{0}\right)} U_{1}^{-1} V_{1} U_{1}^{-1}\right) \\
& =N\left(0, \Sigma_{1}\right) .
\end{aligned}
$$

This completes the proof. 
Proof of Theorem 2 By using Lemma 2 and Lemma 4 instead of Lemma 1 and 3, the proof of this theorem is similar to Theorem 1 , so it is omitted here.

\section{Conclusions}

In this paper, new variable bandwidth nonparametric robust estimators for the drift function and diffusion function of diffusion processes based on discrete-time observations are devised. The new estimators are based on the local linear regression technique and the maximum likelihood type estimation technique, and they have a good control of outliers. The proposed estimators are proved to be consistent and asymptotically normal.

The authors of [23-25] developed robust version estimators of regression function for stationary time series sequence under independent data and dependent data, respectively. Based on their research, in this paper, we studied a continuous-time diffusion process determined by a stochastic differential equation, and our robust estimators based on local linear regression techniques; the reader can consider a robust estimator for a diffusion process by using local polynomial regression techniques. Moreover, the basic ideas of this paper have good generality and can be extended to other continuous-time stochastic models.

In addition, in this paper, we only considered robust estimators for the one dimensional diffusion process; in fact, the basic ideas of our methodology hold for the case of multidimensional diffusion processes and situations.

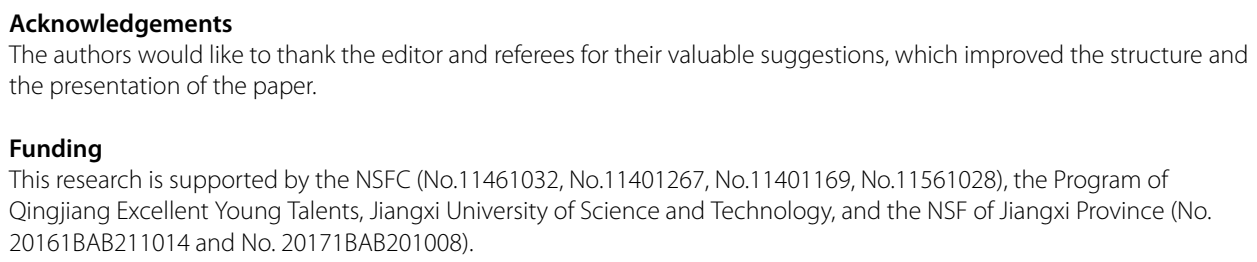

Funding

This research is supported by the NSFC (No.11461032, No.11401267, No.11401169, No.11561028), the Program of Qingjiang Excellent Young Talents, Jiangxi University of Science and Technology, and the NSF of Jiangxi Province (No. 20161BAB211014 and No. 20171BAB201008).

Competing interests

The authors declare that they have no competing interests.

Authors' contributions

All authors jointly worked on the results and they read and approved the final manuscript.

\section{Publisher's Note}

Springer Nature remains neutral with regard to jurisdictional claims in published maps and institutional affiliations.

Received: 10 August 2017 Accepted: 29 January 2018 Published online: 06 February 2018

\section{References}

1. Chaiyapo, N., Phewchean, N.: An application of Ornstein-Uhlenbeck process to commodity pricing in Thailand. Adv. Differ. Equ. 2017, 179 (2017)

2. Zou, T., Chen, S.X.: Enhancing estimation for interest rate diffusion models with bond prices. J. Bus. Econ. Stat. 35 , 486-498 (2017)

3. Tang, C.Y., Chen, S.X.: Parameter estimation and bias correction for diffusion processes. J. Econom. 149, 65-81 (2009)

4. Leonenko, N.N., Šuvak, N.: Statistical inference for student diffusion process. Stoch. Anal. Appl. 28, 972-1002 (2010)

5. Leonenko, N.N., Šuvak, N.: Statistical inference for reciprocal gamma diffusion process. J. Stat. Plan. Inference 140, 30-51 (2010)

6. Li, J., Wu, J.L.: On drift parameter estimation for mean-reversion type stochastic differential equations with discrete observations. Adv. Differ. Equ. 2016, 90 (2016)

7. Nishiyama, Y.: Asymptotic theory of semiparametric Z-estimators for stochastic processes with applications to ergodic diffusions and time series. Ann. Stat. 37, 3555-3579 (2009)

8. Kristensen, D.: Pseudo-maximum likelihood estimation in two classes of semiparametric diffusion models. J. Econom. $156,239-259$ (2010)

9. Koo, B., Linton, O.: Estimation of semiparametric locally stationary diffusion models. J. Econom. 170, 210-233 (2012)

10. Bandi, F., Phillips, P.: Fully nonparametric estimation of scalar diffusion models. Econometrica 71, 241-283 (2003) 
11. Comte, F., Genon-Catalot, V., Rozenholc, Y.: Penalized nonparametric mean square estimation of the coefficients of diffusion processes. Bernoulli 13, 514-543 (2007)

12. Fan, J., Zhang, C.: A re-examination of Stanton's diffusion estimations with applications to financial model validation. J. Am. Stat. Assoc. 98, 118-134 (2003)

13. Xu, K.L.: Empirical likelihood-based inference for nonparametric recurrent diffusions. J. Econom. 153, 65-82 (2009)

14. Xu, K.L.: Re-weighted functional estimation of diffusion models. Econom. Theory 26, 541-563 (2010)

15. Kristensen, D.: Nonparametric filtering of the realized spot volatility: a kernel-based approach. Econom. Theory 26 60-93 (2010)

16. van der Meulen, F., Schauer, M., van Zanten, H.: Reversible jump MCMC for nonparametric drift estimation for diffusion processes. Comput. Stat. Data Anal. 71, 615-632 (2014)

17. Florens-Zmirou, D.: On estimating the diffusion coefficient from discrete observations. J. Appl. Probab. 30, 790-804 (1993)

18. Jiang, G.J., Knight, J.L.: A nonparametric approach to the estimation of diffusion processes, with an application to a short-term interest rate model. Econom. Theory 13, 615-645 (1997)

19. Stanton, R.: A nonparametric model of term structure dynamics and the market price of interest rate risk. J. Finance 52, 1973-2002 (1997)

20. Huber, P.J.: Robust regression: asymptotics, conjectures and Monte Carlo. Ann. Stat. 1, $799-821$ (1973)

21. Delecroix, M., Hristache, M., Patilea, V.: On semiparametric M-estimation in single-index regression. J. Stat. Plan. Inference 136, 730-769 (2006)

22. Davis, R.A., Knight, K., Liuc, J.: M-estimation for autoregressions with infinite variance. Stoch. Process. Appl. 40 145-180 (1992)

23. Fan, J., Jiang, J.: Variable bandwidth and one-step local M-estimator. Sci. China Math. 43, 65-81 (2000)

24. Jiang, J.C., Mack, Y.P.: Robust local polynomial regression for dependent data. Stat. Sin. 11, 705-722 (2001)

25. Cai, Z., Ould-Said, E.: Local M-estimator for nonparametric time series. Stat. Probab. Lett. 65, 433-449 (2003)

26. Wang, Y.Y., Tang, M.T.: Nonparametric robust function estimation for integrated diffusion processes. J. Nonlinear Sci. Appl. 9, 3048-3065 (2016)

27. Øksendal, B.: Stochastic Differential Equations: An Introduction with Applications. Springer, New York (2005)

28. Fan, J., Gijbels, I.: Variable bandwidth and local linear regression smoothers. Ann. Stat. 29, 2008-2036 (1992)

29. Fan, J., Gijbels, I.: Data-driven bandwidth selection in hold polynomial fitting: variable bandwidth and spatial adaption. J. R. Stat. Soc. B 57, 371-394 (1995)

30. Hall, P., Hu, T.C., Marron, J.S.: Improved variable window estimators of probability densities. Ann. Stat. 23, 1-10 (1995)

31. Karatzas, I., Shreve, S.: Brownian Motion and Stochastic Calculus. Springer, New York (2012)

32. Hansen, L., Scheinkman, J.: Back to the future: generating moment implications for continuous-time Markov processes. Econometrica 63, 767-804 (1995)

\section{Submit your manuscript to a SpringerOpen ${ }^{\circ}$ journal and benefit from:}

- Convenient online submission

- Rigorous peer review

- Open access: articles freely available online

- High visibility within the field

- Retaining the copyright to your article

Submit your next manuscript at $\gg$ springeropen.com 